\title{
Warm $\mathrm{C}_{2} \mathrm{H}_{2}$ toward NGC 7538 IRS9: Grain Surface Origin
}

\author{
Steven D. Doty'1, Sandra L. Doty², Jeff Cochran1, John Lacy ${ }^{3}$, John Barentine ${ }^{3}$, Richard Field1 \\ ${ }^{1}$ Department of Physics and Astronomy, Denison University, Granville, USA \\ ${ }^{2}$ Department of Physics, Ohio University Lancaster, Lancaster, USA \\ ${ }^{3}$ Department of Astronomy, University of Texas, Austin, USA \\ Email: doty@denison.edu
}

Received 28 June 2014; revised 20 July 2014; accepted 15 August 2014

Copyright (C) 2014 by authors and Scientific Research Publishing Inc.

This work is licensed under the Creative Commons Attribution International License (CC BY).

http://creativecommons.org/licenses/by/4.0/

(c) (i) Open Access

\section{Abstract}

We consider models for the observed ro-vibrational absorption lines of acetylene toward NGC 7538 IRS9. The data are fit with multiple screens, each having separate column densities, rotational and vibrational excitation temperatures, and filling factors. The best fit was determined using a chi-squared minimization scheme. We find that only one screen is necessary-multiple screens gave rise to either making one of the screens transparent, or very occasionally making the two screens the same. As a result, we can place constraints on $T_{\text {rot }}, T_{\text {vib }}, \mathbf{N}_{\mathrm{C} 2 \mathrm{H} 2}$, and the filling factor, f. In particular we find $0.03<\mathrm{f}<0.3$ with a best fit of $\mathrm{f} \sim 0.1$. We also find $\mathrm{T}_{\mathrm{vib}}<200 \mathrm{~K}$, with a best fit of $\mathrm{T}_{\mathrm{vib}}<20 \mathrm{~K}$. We find $\mathrm{N}_{\mathrm{C} 2 \mathrm{H} 2}=2.4+/-0.6 \times 10^{16} \mathrm{~cm}^{-2}$, or that $\mathrm{N} \times \mathrm{f} \sim 2 \times 10^{15} \mathrm{~cm}^{-2}$. Lastly, we find 80 $<T_{\text {rot }}<140 \mathrm{~K}$, with a best fit of $T_{\text {rot }} \sim 100 \mathrm{~K}$. Physically, this can be interpreted as: (1) no vibrational excitation, (2) the warm region only fills a small fraction of the beam, (3) the $\mathrm{C}_{2} \mathrm{H}_{2}$ arises very near a region of $100 \mathrm{~K}$. Chemically, this is in consistent with a model where the $\mathrm{C}_{2} \mathrm{H}_{2}$ is formed in the gas phase. It is however consistent with a scenario where the $\mathrm{C}_{2} \mathrm{H}_{2}$ is evaporated at $100 \mathrm{~K}$ from the grain surface, suggesting either a grain-surface origin or earlier origin followed by condensation. Finally, the $\mathrm{C}_{2} \mathrm{H}_{2}$ column density is consistent with a disk geometry.

\section{Keywords}

Stars: Formation, Stars: High Mass, ISM: Molecules, Molecular Processes, Stars: Individual (NGC 7538 IRS9)

\section{Introduction}

The process by which massive stars form remains uncertain after much study. Generally, two broad models exist. In the first - the core accretion model [1]-[3]—-massive stars from through the accretion of turbulently supported 
gas to form massive proto stars. In the second—the competitive accretion/collision model [4]-[6]—-low mass stars form in clusters, and run-away accretion and stellar collisions lead to a buildup of massive stars.

The envelopes of massive star-forming regions have been well-studied both observationally, and theoretically. While the properties of the outer envelope have given some insight into the microphysics of these regions, two observational effects have limited the ability to infer much about the star-formation process. First, the relative infrequency of massive stars leads to the result that many high-mass star-forming regions lie at large distances (> $1-2 \mathrm{kpc}$ ), and so are not well-resolved. Second, their high opacities during the formation phase, followed by the quick disruption of their natal environment upon "turn-on" makes direct observation of their inner structures difficult at best.

Moving in from the envelope, it is expected that signatures of the star-formation process might well be apparent in the Warm Inner Transition regions (WITs) of these massive star-forming regions. Intuitively, one would expect different distributions of material in these regions. In the collision case, disruption might be expected to either evacuate the disk region and/or produce significant clumping. Indeed, [7] suggests that massive stellar encounters should limit disks to less than 30 AU. Likewise, in the case of core accretion, one might expect disks and large amounts of gas near the protostellar surface. In this vein, [3] predict that massive stars have disks $\sim 100 \mathrm{~s}-1000 \mathrm{~s}$ AU in size.

In this paper, we study the WIT of NGC 7538 IRS9 using mid-infrared (MIR) absorption lines of $\mathrm{C}_{2} \mathrm{H}_{2}$ as observed by TEXES on GEMINI. The MIR lines have three significant advantages in this context. First, the MIR lines are essentially limited in angular resolution only by the size of the warm region against which the absorption occurs, thus more directly probing the WIT. Second, the MIR lines have the potential to probe much higher excitation conditions than low-lying radio line, providing a cut in "excitation conditions" to probe the WIT. Finally, MIR lines provide high data density, so that a large dynamic range of conditions may be probed.

In this paper, the source is discussed in Section 2. The model is then described and the approach to fitting the data is also discussed in Section 3. The models are applied to the data and the results are in Section 4. We discuss the results and their interpretation in Section 5. Finally, Section 6 contains a summary of the results.

\section{NGC 7538 IRS9: Observations and Data}

Reference [8] used trigonometric parallax to place NGC 7538 at approximately $2.7 \mathrm{kpc}$. As such, it is a relatively nearby high-mass star-forming region, and thus a good target for studying the chemical and physical structures involved in high-mass star formation. While the precursors to complex molecules have been observed in the exterior of the circumstellar envelope [9], observations of hot complex molecules are more lacking [10]. As a result, the observations detailed here involve the ro-vibrational absorption lines from $\mathrm{C}_{2} \mathrm{H}_{2}$. Due to the combination of consideration of ro-vibrational lines and the effectively small beam size of absorption measurements against a hot central source, it is expected that these observations may more easily concentrate on the warm interior of NGC 7538 IRS9.

The observations considered here were taken with the Texas Echelon Cross (X) Echelle Spectrograph (TEXES; [11]) on the Gemini North 8m telescope in October 2007, and at the NASA Infrared Telescope Facility (IRTF) in June 2001.The data were originally presented in [12], and are only briefly described here.

The data were obtained in a 0.5 " slit width (4" slit length) on Gemini. The telescope was nodded by 1" - 5", and the adjacent nod positions were subtracted. Sky calibration was carried out in accord with [11], and flux calibration followed the approach of [13].

Acetylene $\left(\mathrm{C}_{2} \mathrm{H}_{2}\right)$ was observed in the v5 R-branch $(\mathrm{J}=5.6)$ as well as approximately ten lines of $v 5$ Q-branch. The $v 5 \mathrm{R}(1)$ and $\mathrm{R}(13)$ lines were observed with IRTF.

Acetylene was chosen as a target molecule due to predictions [14] that it could be produced in the gas phase

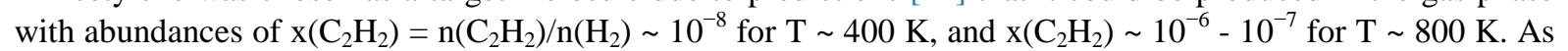
a result, gas-phase production of $\mathrm{C}_{2} \mathrm{H}_{2}$ would be a signature of warm gas, expected to arise from the central WIT of NGC 7538 IRS9.

\section{Model Description and Fitting Approach}

\subsection{Model Description}

The most exact model that one might construct would involve a non-local treatment including the simultaneous 
solution of both the equations of statistical equilibrium and radiative transfer. This has been done by many authors (e.g. [15]-[17]). In this application, we are hampered by two problems. First, due to the fact that these are rovibrational lines, a very large number of lines must be considered. This makes the problem extremely CPU intensive, thus significantly reducing the ability to constrain the source properties. Second, and most importantly, data do not exist for the rovibrational collision rates.

With these two difficulties, we take a separate approach. Given that the lines are seen in absorption, we consider instead absorbing screens between the observer and the background against which the absorption occurs. In this approach, we aim to understand the distribution of material that contributes to the absorption. In particular, we wish to understand 1) how much material does the absorption, 2) how distributed in excitation is the material, and 3) how extended is the material/how much of the emitting background is covered by the absorbing material. Once these gross properties of the absorption are understood, it will be possible to see to what extent these macroscopic effects are (in) consistent with various geometries and excitation/production mechanisms.

Each of the absorbing screens is characterized by four main factors: column density of $\mathrm{C}_{2} \mathrm{H}_{2}\left(\mathrm{~N}_{\mathrm{i}}\right)$, filling factor $\left(f_{i}\right)$, rotational excitation temperature $\left(T_{\text {rot, }, i}\right)$, and vibrational excitation temperature $\left(T_{\text {vib,i }}\right)$. Here the index corresponds to the screen number, so that each screen is allowed to have its own combination of properties. While the radial and turbulent velocities are also parameters for each screen, they are fixed at $\mathrm{v}_{\mathrm{r}}=0$ and $\mathrm{v}_{\mathrm{t}}=3$ $\mathrm{km} \cdot \mathrm{s}^{-1}[18]$.

Once the screen properties are defined, we are able to define the level populations for the screens. The $\mathrm{C}_{2} \mathrm{H}_{2}$ level assignments, energies, transitions, and Einstein A coefficients are taken from the GEISA database [19]. The background source is taken to be a blackbody, though due to the background subtraction, the details of the background do not affect the modeled lines. Given the background and molecular information for the screens, we solve the radiative transfer equation by ray-tracing from the background source, through the screens, to the observer.

\subsection{Fitting}

The quality of fit between the simulated and actual observations are calculated through a reduced $\chi^{2}$, namely,

$$
\chi^{2}=\frac{1}{\mathrm{~N}} \sum_{\mathrm{i}=1}^{\mathrm{N}} \frac{\left(\mathrm{y}_{\mathrm{i}, \mathrm{obs}}-\mathrm{y}_{\mathrm{i}, \mathrm{mod}}\right)^{2}}{\sigma_{\mathrm{i}}^{2}}
$$

In this expression, $\mathrm{N}$ is the number of spectral channels being summed over, $\chi_{\mathrm{i}}$ is the uncertainty in the $\mathrm{i}^{\text {th }}$ datapoint, and $\mathrm{y}_{\mathrm{i}, \text { obs }}$ and and $\mathrm{y}_{\mathrm{i} \text {,mod }}$ are the observed and modeled data for the $\mathrm{i}^{\text {th }}$ point respectively. Data points that do not lie within a line have the effect of artificially lowering the $\chi^{2}$ value. As a result, we limit our fits to the regions near the lines. We also remove any extraneous points with atmospheric transmittances below 0.25 . As these definitions are ad hoc, there is some flexibility in the actual values of the computed $\chi^{2}$. However, these assumptions do not change the qualitative nature of the $\chi^{2}$, nor do they change the location of the minimum (bestfit) in parameter space.

In doing the fitting, we employ two approaches. In the first, we do a global fitting. Starting with an initial guess for each of the parameters, we use the Levenberg-Marquardt algorithm as expressed in DNLS1 of the MINPACK library [20]. In order to consider the possibility that the parameter space maybe topologically complicated, we start the fitting multiple (over 1000) times. We compare the best fits from each start. We find that almost every start leads to the same result, to within numerical noise. We call the final solution with the lowest $\chi^{2}$ the best fit.

In our second approach to the fitting, we construct a grid of models. Starting from the best fit above, we modify the input parameters in a regular fashion to obtain $\chi^{2}$ values for models in the neighborhood of the best fit. This gridding approach allows us to 1) confirm the validity of the best fit in the local region, and 2) understand the topology of the local parameter space. This second point not only aids in our understanding of the data, but more importantly helps to provide confidence intervals on our results in the next section.

\section{Results}

\subsection{Multiple-Screen Best Fits}

In our first approach to fitting, we considered two screens, each having their own column density, and rotational 
and vibrational excitation temperatures. The results of the four best fits are shown in Table 1 . In the four best fits (from our initial list of $\sim 1000$ different initial conditions), we find a mean ratio of column densities between the two screens of $\mathrm{N}_{1} / \mathrm{N}_{2}=292$. In these fits, one of the screens is transparent and plays little-to-no role in the absorption. These fits are statistically indistinguishable, having $\chi^{2}=0.91+/-0.01$. Further supporting this idea is near constancy of the rotational temperature, and consistently low value of the vibrational temperature.

The best fit is shown in Figure 1. As can be seen, those data with the highest signal-to-noise ratio (SNR) are fit quite well. As the SNR decreases, the fits still generally match the depth and position of the observed lines, though the noisiest data (the Q-branch) are quite difficult to consider due to instrumental/baseline effects. To show the effect of various values of $\chi^{2}$ in Figure 2, we plot three different fits. The solid line corresponds to the best fit having $\chi^{2}$ bf $=0.91$. The long-dashed line corresponds to $\chi^{2}=1.1 \chi_{b f}^{2}=1.0$. Finally, the dotted line is for a model with $\chi^{2}=1.5$. From this we see that as defined here, a $\chi^{2}$ value of 1.0 provides an adequate fit, but one still observably different from the best fit. By the time $\chi^{2}=1.5$, we find generally unacceptable fits. As a result, in the following discussions, we consider $\chi^{2}<1$ to be acceptable and $\chi^{2}>1.0$ to be not acceptable.

\subsection{Two-Screen Grids: Confirming One Screen}

To understand the robustness of the result above that one screen dominates the absorption, we have constructed grids of two-screen models based around the best fit properties. In particular, we can consider what happens when the column densities of the two screens are varied.

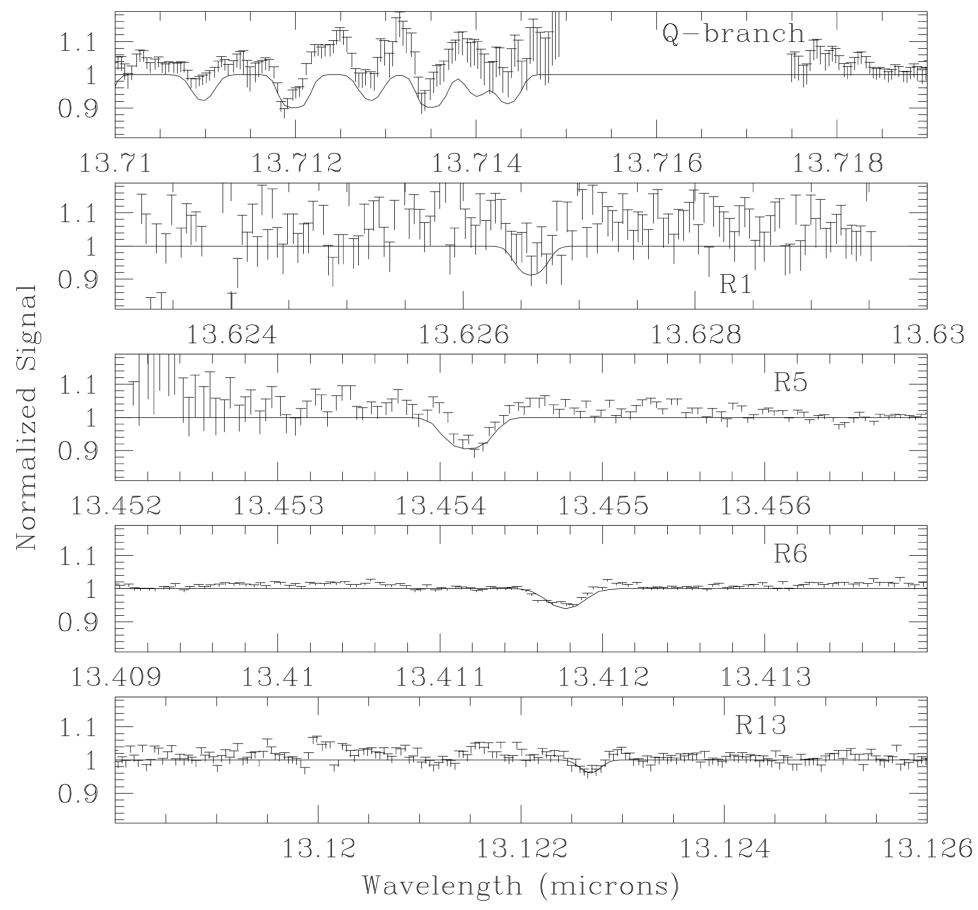

Figure 1. The observed data (points with error-bars) and best fit model (solid lines) for $\mathrm{C}_{2} \mathrm{H}_{2}$ toward NGC 7538 IRS 9.

Table 1. Sample of four best fit two-screen models.

\begin{tabular}{ccccc}
\hline Fit & $\mathrm{N}_{1} / \mathrm{N}_{2}$ & $\mathrm{~T}_{\text {rot, } \mathrm{i}}$ & $\mathrm{T}_{\text {vib,i }}$ & $\chi^{2}$ \\
\hline 1 & 355 & 143 & 28 & 0.907 \\
2 & 718 & 143 & 44 & 0.913 \\
3 & 44 & 123 & 50 & 0.915 \\
4 & 53 & 143 & 81 & 0.918 \\
\hline
\end{tabular}




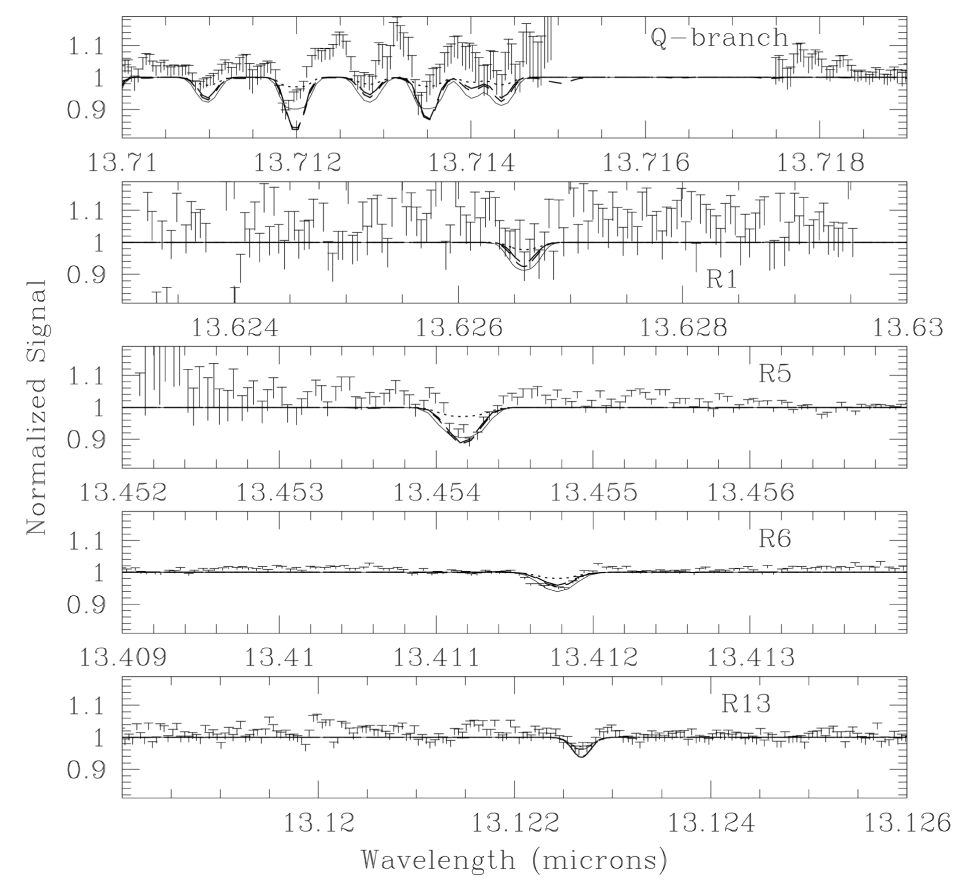

Figure 2. The observed data (points with error bars) and different fits. The best fit $\left(\chi^{2}=0.91\right)$ is the solid line. The long dashed line has $\chi^{2}=1.0$, and the dotted line has $\chi^{2}=1.5$ for comparison.

The results of this approach are shown in Figure 3. As can be seen, the results are degenerate, namely only one total column density is consistent with the data, $\mathrm{N}\left(\mathrm{C}_{2} \mathrm{H}_{2}\right)=2.4+/-0.6 \times 10^{16} \mathrm{~cm}^{-2}$. It is important to note that in these grids, the column densities were fixed at each point on the grid, while the excitation temperatures were allowed to vary. In these cases, the excitation temperatures were nearly always within a few percent of each other.

The final conclusion is that, as noted previously, the absorption appears to be dominated by a single screen of material.

\subsection{One-Screen Grids: Determining Material Properties}

With the determination that most of the absorbing material lies in one screen, it is possible to better understand the topology of the parameter space around that best fit. By understanding the topology we can better understand the robustness of the results, and place uncertainty limits on our derived quantities.

To do this, we consider grids of single-screen models around the best-fit result. The results are shown in Figures 4-6. From these figures, it is clear that it is possible to constrain the screen properties with meaningful precision. In particular, we see that the column densities lie in the range $1.8 \times 10^{16}<\mathrm{N}\left(\mathrm{C}_{2} \mathrm{H}_{2}\right)<3.0-10^{16}$. The vibrational excitation temperature lies in the range $\mathrm{T}_{\mathrm{vib}}<200 \mathrm{~K}$. And finally, the rotational excitation temperature lines in the range $80<\mathrm{T}_{\text {rot }}<140$.

We can also see the effect of varying the filling factor. In Figure 7 we plot the model results (along with their uncertainties) as a function of filling factor. Here we assume four filling factors, $0.03,0.1,0.3$, and 1.0. As can be seen from the top panel, the best fit—signified by the minimum $\chi^{2} / \chi^{2}$ min occurs at $\mathrm{f}=0.1$. This is consistent with our previous results.

Interestingly, the vibrational temperature in all cases is low $\left(\mathrm{T}_{\mathrm{vib}}<200 \mathrm{~K}\right)$. Even more importantly, it is always consistent with $\mathrm{T}_{\mathrm{vib}}=0 \mathrm{~K}$, when the uncertainties are considered.

Finally, even though the filling factor varies, the "total column density" as measured by $\mathrm{N}_{(}\left(\mathrm{C}_{2} \mathrm{H}_{2}\right) \times \mathrm{f}$ is always consistent with $\mathrm{N} \times \mathrm{f} \sim 1.5-2.5 \times 10^{15} \mathrm{~cm}^{-2}$. In other words, the total number of $\mathrm{C}_{2} \mathrm{H}_{2}$ molecules along the line of sight integrated over the background emitting region is independent of the filling factor adopted in the modeling. 


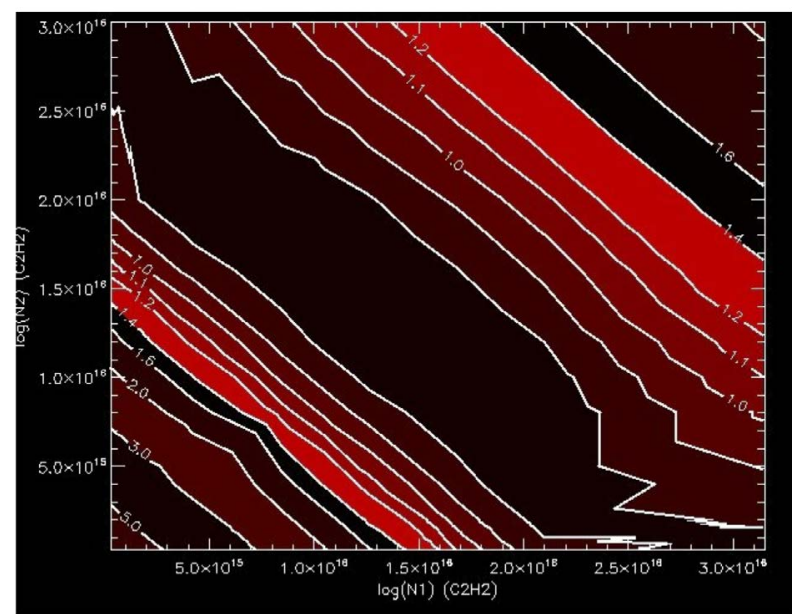

Figure 3. Contours of $\chi^{2}$ for two screens, as a function of the column densities of each of the screens. Notice the degeneracy, such that the two screens act as one.

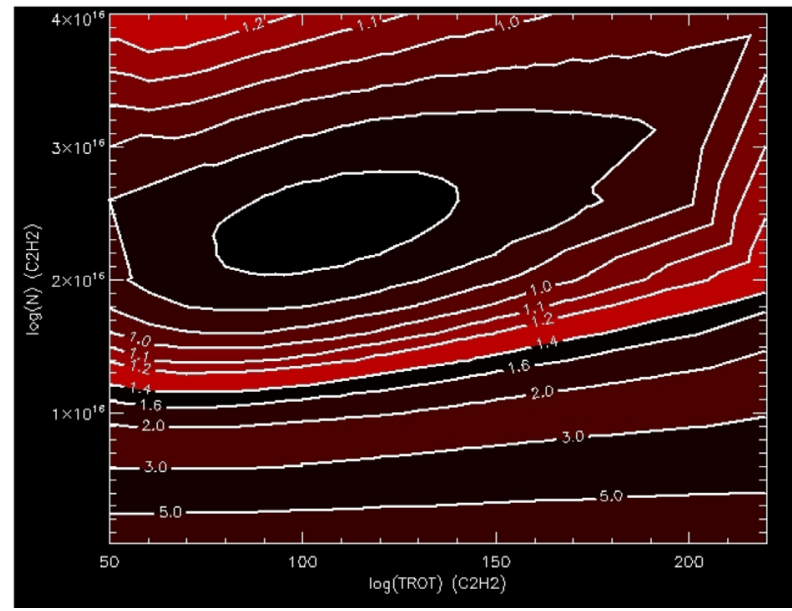

Figure 4. Contours of $\chi^{2}$ for one screen, as a function of the column density and rotational temperature of the screen.

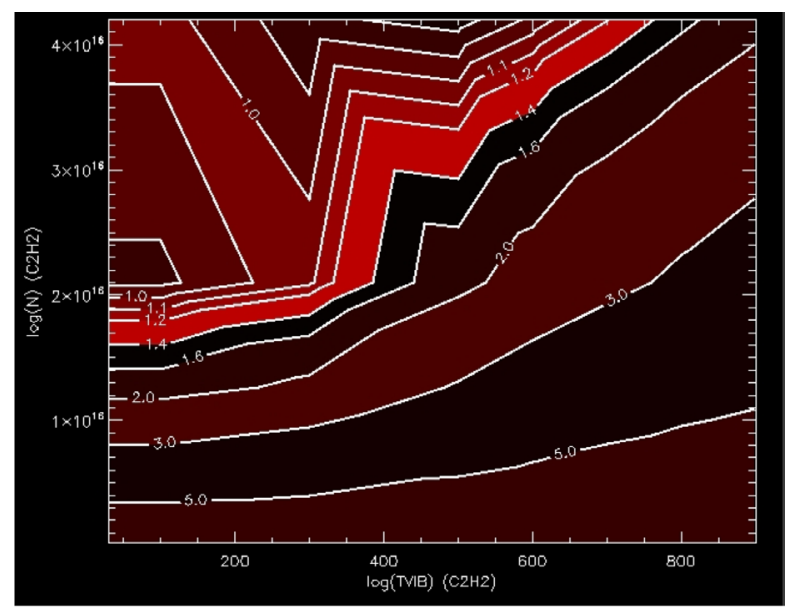

Figure 5. Contours of $\chi^{2}$ for one screen, as a function of the column density and vibrational temperature of the screen. 


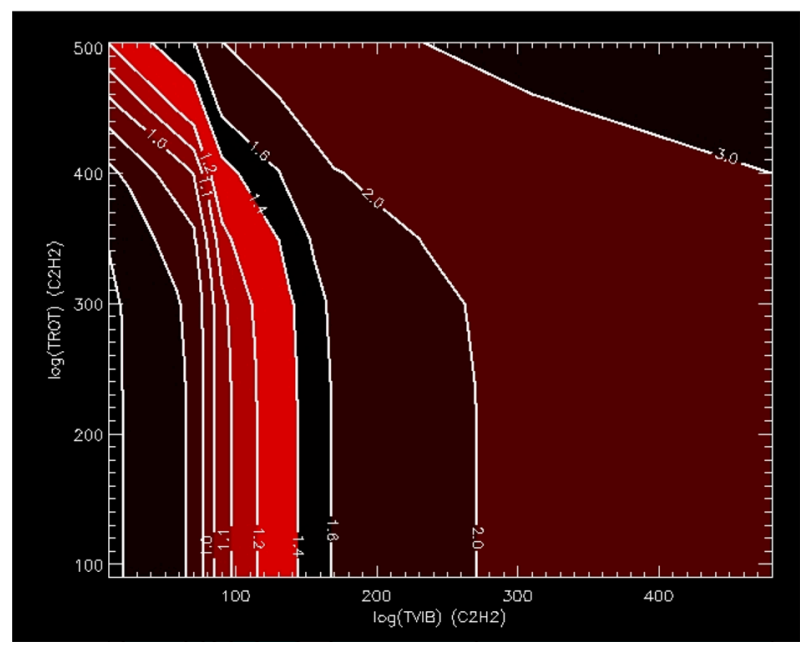

Figure 6. Contours of $\chi^{2}$ for one screen, as a function of the rotational and vibrational temperatures of the screen.

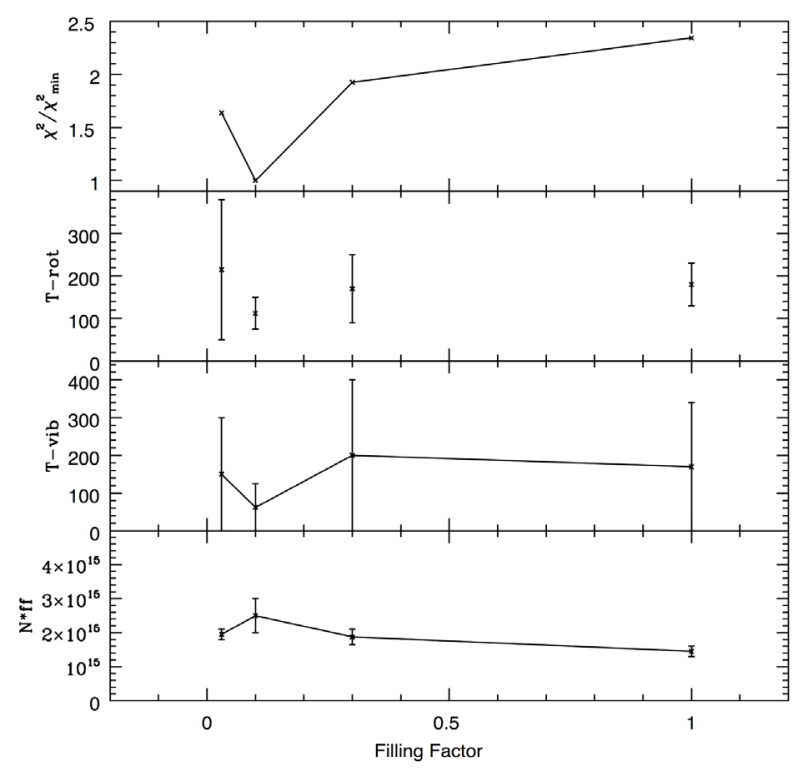

Figure 7. The dependence of various physical parameters on the filling factor. The top panel shows $\chi^{2}$ relative to the minimum value. The second and third panels show the rotational and vibrational excitation temperatures. The bottom panel shows the total amount of $\mathrm{C}_{2} \mathrm{H}_{2}$ covering the source, as measured by the column density (in units of $\mathrm{cm}^{-2}$ ) times the filling factor. The error bars on each point are limits on the uncertainty of the data from the fitting.

\section{Discussion}

The best-fit parameters for the absorbing screen toward NGC 7538 IRS9 from the previous section are shown in Table 2. From these parameters, we seek to infer the origin of the $\mathrm{C}_{2} \mathrm{H}_{2}$, as well as understand what constraints it may place on the structure of the WIT contained in NGC7538 IRS9.

\subsection{Origin of $\mathrm{C}_{2} \mathrm{H}_{2}$}

One of the most interesting results of the modeling is the rotational excitation temperature of the $\mathrm{C}_{2} \mathrm{H}_{2}$, namely $\sim 100 \mathrm{~K}$ (actually $80-140 \mathrm{~K}$ ). The photon reprocessing due to the high optical depths, together with the dust- 
Table 2. Best Fit Parameters for Absorbing $\mathrm{C}_{2} \mathrm{H}_{2}$ Screen Toward NGC 7538 IRS 9.

\begin{tabular}{cc}
\hline Parameter & Best Fit Region \\
\hline Column Density $\left[\mathrm{N}\left(\mathrm{C}_{2} \mathrm{H}_{2}\right)\right]$ & $2.0-2.6 \times 10^{16} \mathrm{~cm}^{-2}$ \\
Rotational Temperature $\left[\mathrm{T}_{\text {rot }}\right]$ & $80-140 \mathrm{~K}$ \\
Vibrational Temperature $\left[\mathrm{T}_{\text {vib }}\right]$ & $<100 \mathrm{~K}$ \\
Filling Factor [f] & $0.1(0.03<\mathrm{f}<0.3)$ \\
\hline
\end{tabular}

modified Wein's Law from [21] yields a dust temperature emitting in this spectral range of $\mathrm{T}>250 \mathrm{~K}$. As a result, the background source appears to be spatial separate from the absorbing material.

While the physical location of the absorbing screen relative to the background source is to be expected, the rotational excitation temperature is something of a surprise since the gas-phase modeling of hot cores by [14] and the recent results of [22] suggest that $\mathrm{C}_{2} \mathrm{H}_{2}$ does not reach fractional abundances of $10^{-7}-10^{-8}$ in the gas-phase until at least $400-800 \mathrm{~K}$. At the temperatures of $\mathrm{T} \sim 100 \mathrm{~K}$ here, [22] predicts fractional abundances of $<10^{-9}$. This is shown in Figure 8, where we plot fractional abundance contours as a function of the radiation field, specified as $G_{0}$, the ratio of the UV radiation field relative to the standard interstellar radiation field. At this fractional abundance, an $\mathrm{H}_{2}$ column density of $10^{26} \mathrm{~cm}^{-2}$ is required to achieve the observed $\mathrm{C}_{2} \mathrm{H}_{2}$ column density. This is much larger than the inferred $\mathrm{H}_{2}$ column density of $\sim 5 \times 10^{22} \mathrm{~cm}^{-2}$ [23] [24].

Taken together, the data above argue significantly against a gas-phase origin for $\mathrm{C}_{2} \mathrm{H}_{2}$ in this absorption screen. Given the fact that water ice is observed to sublimate at 100 K [25], the excitation temperature of 80 $140 \mathrm{~K}$ suggests a grain-surface origin. Indeed, [26] [27] find that many species are trapped in the water ice in grain mantles, and co-desorb with water near $100 \mathrm{~K}$. The range of temperatures in the $100-140 \mathrm{~K}$ range is reasonable, and may be due to differences in the type of ice, kinematics if the material is in falling, or reprocessing of the $\mathrm{C}_{2} \mathrm{H}_{2}$ at higher temperatures.

From a theoretical perspective, formation of $\mathrm{C}_{2} \mathrm{H}_{2}$ is reasonable. As early as [28], abundances of $\mathrm{C}_{2} \mathrm{H}_{2}$ as high as $\mathrm{x} \sim 10^{-7}$ have been predicted on grain surfaces. Cometary measurements [29] and laboratory work [30] suggest that $\mathrm{C}_{2} \mathrm{H}_{2}$ should exist as a component in ices. On the other hand, [31] makes the suggestion that AGB stars form $\mathrm{C}_{2} \mathrm{H}_{2}$ as precursors to grains, and that the remaining $\mathrm{C}_{2} \mathrm{H}_{2}$ freezes onto the grain surface.

While we cannot say with any certainty what the origin of the $\mathrm{C}_{2} \mathrm{H}_{2}$ is in the ices-whether they are formed in the gas phase and adsorbed, or whether they are formed on the ice mantles themselves-it does appear that $\mathrm{C}_{2} \mathrm{H}_{2}$ is liberated from the ice mantles toward NGC 7538 IRS9.

This suggestion is perhaps consistent with NGC 7538 IRS1, a separate high-mass YSO in this region. Reference [32] finds that a consistent model for $\mathrm{HCN}$ and $\mathrm{C}_{2} \mathrm{H}_{2}$ cannot be achieved from gas-phase chemistry alone. They suggested that $\mathrm{C}_{2} \mathrm{H}_{2}$ evaporation from icy mantles might explain their observations.

\subsection{Pumping of $\mathrm{C}_{2} \mathrm{H}_{2}$ by Radiation}

It appears that there is little if any radiative pumping or excitation of $\mathrm{C}_{2} \mathrm{H}_{2}$ by radiation. This can be seen from the inferred vibrational excitation temperatures. While we find $\mathrm{T}_{\text {vib }}<100 \mathrm{~K}$, all results appear to be consistent with $\mathrm{T}_{\mathrm{vib}} \sim 0 \mathrm{~K}$. This result is probably not a surprise, given the relatively large $\mathrm{H}_{2}$ column density $\sim 5 \times 10^{22}$ $\mathrm{cm}^{-2}$ toward NGC 7538 IRS9. As a result, the $\sim 100 \mathrm{~K} \mathrm{C}_{2} \mathrm{H}_{2}$ observed here would see little if any of the $\sim 6 \mu \mathrm{m}$ radiation that would be necessary to excite the higher vibrational levels and thus populate the upper level probed here.

\subsection{Possible Implication for the WIT Geometry}

It is interesting to speculate about the implications for the WIT geometry in NGC 7538 IRS9 based upon these results. There are four that we choose to consider here: sphere, clumps, outflow cavity wall, and central disk. In considering these possible geometries, we will take the beam as slightly larger than the diffraction-limited size $\sim 1400 \mathrm{AU}$ at the adopted distance of $2.8 \mathrm{kpc}$.

The first case to consider is the spherical geometry. Dust emission from NGC 7538 IRS9 was observed and modeled by [33]. They found a central source of $\mathrm{L}=3.6 \times 10^{4} \mathrm{~L}_{\text {sun }}$, density power law index $\mathrm{p}=1.75$, and den- 


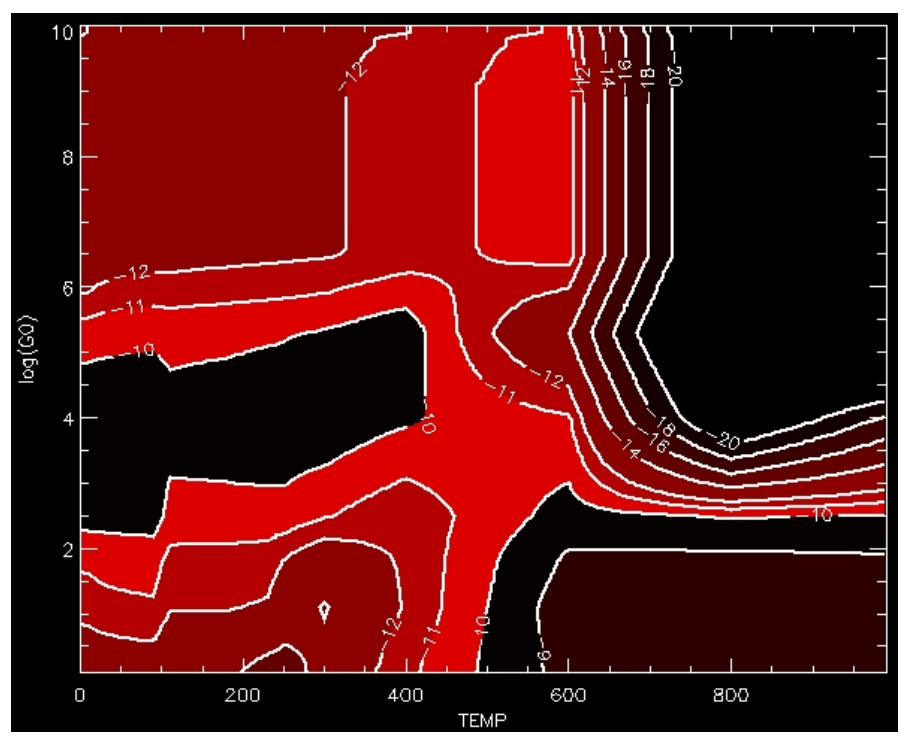

Figure 8. Contours of $\mathrm{C}_{2} \mathrm{H}_{2}$ abundance as a function of the radiation field $\left(\mathrm{G}_{0}\right)$ and temperature.

sity at $\mathrm{r}=1000 \mathrm{AU}$ of $\mathrm{n}_{1000}=1.2 \times 10^{7} \mathrm{~cm}^{-3}$. Such a spherical model generates a dust temperature of $100 \mathrm{~K}$ at $\mathrm{r}$ $\sim 2700 \mathrm{AU}$. As a result, the absorbing screen would fill approximately the entire beam-a result inconsistent with the inferred beam-filling factor $\mathrm{f}=0.1$.

If the general star-forming region is somewhat regular on large scales, but not on all scales as shown above, it is possible that the WIT is clumpy due to fragmentation or outflows. In this case, the $\mathrm{C}_{2} \mathrm{H}_{2}$ could be scattered through the WIT in clumps. If we assume that clumps should be no larger than about one-tenth the size of the beam, this would yield $\mathrm{r}_{\text {clump }}<10^{15} \mathrm{~cm}$. If we allow a clump to have a number density of $\mathrm{n}_{\text {clump }}=10^{7} \mathrm{~cm}^{-3}$, we find that the relative $\mathrm{C}_{2} \mathrm{H}_{2}$ abundance necessary to achieve the inferred column density of $2.5 \times 10^{16} \mathrm{~cm}^{-2}$ is $\mathrm{x}$ $\left(\mathrm{C}_{2} \mathrm{H}_{2}\right)>2.5 \times 10^{-6}\left(\mathrm{~N} / 2.5 \times 10^{16}\right)\left(10^{7} / \mathrm{n}_{\text {lump }}\right)\left(10^{15} / \mathrm{r}_{\text {clump }}\right)$. This is a lower limit to the $\mathrm{C}_{2} \mathrm{H}_{2}$ fractional abundance, and is approximately 25 - 100 times greater than that found either in the gas-phase [14] [22] or solid-phase predictions [28].

A third possibility, raised by results for the high-mass source AFGL 2591 [34]-[36], is an out flow cavity wall subject to irradiation from the central source. Reference [37] suggests that the source is nearly pole-on, as observed by bipolar, extremely high velocity out flows. Since the outflow walls will presumably extend to the edge of the envelope, the cavity will fill a significant portion of the beam, and more probably fill it completely. This is much greater than the inferred filling factor, and thus rules out the outflow cavity wall scenario.

It is also possible that the $\mathrm{C}_{2} \mathrm{H}_{2}$ ice may be transported via in falling filaments observed toward massive star-forming regions [38] [39]. These regions show evidence of complex molecules and in fall on spatial scales down to approximately 5000 - 10,000 AU. Unfortunately, the temperatures observed toward these regions may be somewhat high (up to $200 \mathrm{~K}$ [39]) compared to the $\mathrm{C}_{2} \mathrm{H}_{2}$ temperatures inferred here.

The final case which we may consider is a massive disk. To be clear, this is speculative since disk around massive YSOs have not been directly observed. However, simulations (e.g. [40]) suggest that disk-like structures may be formed, and observations (e.g. [41]) have found disk candidates around high-mass YSOs. Disks around high-mass stars may be expected to be approximately $\mathrm{r} \sim 400 \mathrm{AU}$ (e.g. [42]). If we take a distance $\mathrm{l}=100$ AU over which $\mathrm{C}_{2} \mathrm{H}_{2}$ exists, we find a required $\mathrm{C}_{2} \mathrm{H}_{2}$ fractional abundance of $x\left(\mathrm{C}_{2} \mathrm{H}_{2}\right)=10^{-9}\left(1.5 \times 10^{15} / \mathrm{l}\right)$. This would be reasonable, with sufficient range to allow for different production conditions/mechanisms.

The second issue to consider with disks is the beam-filling factor. Taking the beam-filling factor to be the area of the beam filled by $\mathrm{C}_{2} \mathrm{H}_{2}$ divided by the total area of the beam, this can be easily calculated. A beam size of $700 \mathrm{AU}$ would suggest a $\mathrm{C}_{2} \mathrm{H}_{2}$ region of size $200 \mathrm{AU}$ to achieve $\mathrm{f}=0.1$. For comparison, in Figure 9 we plot the temperature distribution for a high-mass disk using the Pro Dimo code [43]. Note that the region including $\mathrm{T} \sim$ $100 \mathrm{~K}$ extends out to $\mathrm{r} \sim 200 \mathrm{AU}$, and has a vertical thickness of approximately $\Delta \mathrm{z} \sim 100 \mathrm{AU}$. While such highmass disks are not certain, it is interesting to note that such a disk would be consistent with the observations. This possibility, and the potential role of filaments, will be the subject of a following paper. 


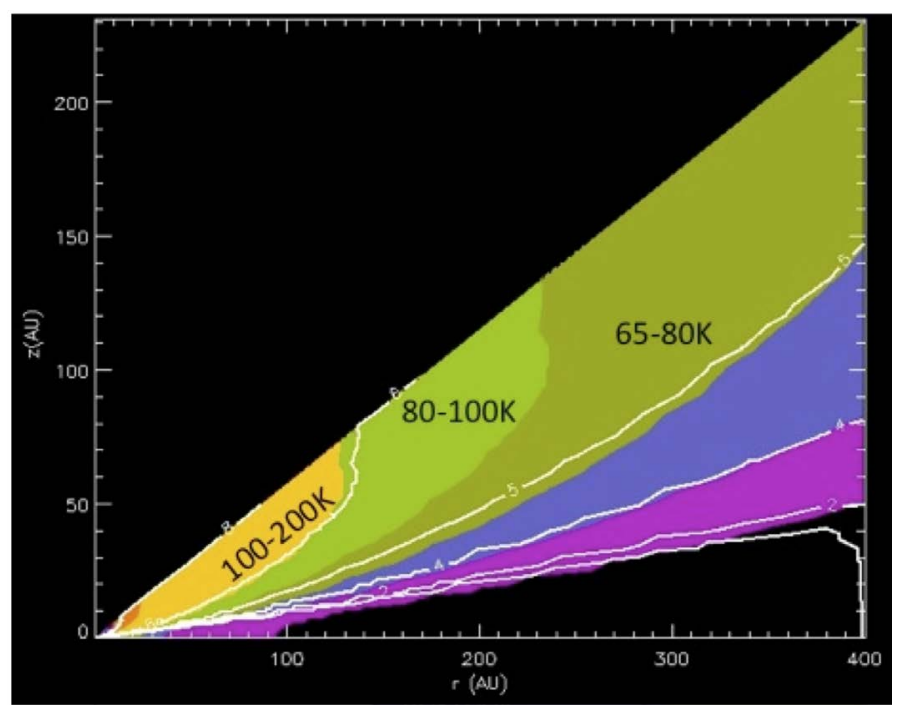

Figure 9. The temperature distribution in a sample high-mass disk as calculated using the ProDimo code. Note the region of T $100 \mathrm{~K}$ extends to approximately $200 \mathrm{AU}$.

\section{Conclusions}

In this paper we have described the observation, modeling, and interpretation of ro-vibrational absorption lines of $\mathrm{C}_{2} \mathrm{H}_{2}$ toward the high-mass star-forming region NGC 7538 IRS9. The goal is to understand the conditions of the warm, inner, transition (WIT) that exists between the relatively regular envelope and the protostar. We have performed fits on the data where we vary the rotational and vibrational excitation temperatures, the filling factor, and the $\mathrm{C}_{2} \mathrm{H}_{2}$ column density. We followed these fits up with local grids to understand the topology of the surrounding parameter space, as well as the robustness of the inferred source parameters. The resulting interpretation can be concluded as follows:

1) Even when multiple components are allowed, the data are degenerately best fit by a single screen, suggesting that the gas-phase $\mathrm{C}_{2} \mathrm{H}_{2}$ has primary dominant origin (Section 4.1).

2) The best fit model properties include: a column density $\mathrm{N}\left(\mathrm{C}_{2} \mathrm{H}_{2}\right)=2.4+/-0.6 \times 10^{16} \mathrm{~cm}^{-2}$, a rotational excitation temperature of $80<\mathrm{T}_{\text {rot }}<140 \mathrm{~K}$, avibrational temperature is $\mathrm{T}_{\text {vib }}<200 \mathrm{~K}$ (and consistent with $\mathrm{T}_{\text {vib }}=0$ ), and a filling factor is $\mathrm{f}=0.1$ (Sections $4.2 \& 4.3$ ).

3) The column density, and excitation temperature are both in consistent with a gas-phase origin for $\mathrm{C}_{2} \mathrm{H}_{2}$ (Section 5.1).

4) While it is unclear if the $\mathrm{C}_{2} \mathrm{H}_{2}$ is formed on the grain surfaces or freezes out there from the gas phase at some earlier time, the $\sim 100 \mathrm{~K}$ rotational temperature is interpreted as due to grain-surface evaporation of $\mathrm{C}_{2} \mathrm{H}_{2}$ (Section 5.1).

5) The low vibrational temperature is consistent with effectively no infrared radiative pumping of higher lying vibrational lines (Section 5.2).

6) The data are not consistent with a spherical, clumping, or outflow cavity wall for the WIT. However, a high-mass disk is consistent with the observations (Section 5.3).

\section{Acknowledgements}

This work was supported by NASA Grant NNX08AH28G (SDD) and by a research release (SLD).

\section{References}

[1] McKee, C.F. and Tan, J.C. (2002) Massive Star Formation in 100,000 Years from Turbulent and Pressurized Molecular Clouds. Nature, 416, 59-61. http://dx.doi.org/10.1038/416059a

[2] McKee, C.F. and Tan, J.C. (2003) The Formation of Massive Stars from Turbulent Cores. The Astrophysical Journal, 585, 850. http://dx.doi.org/10.1086/346149 
[3] Krumholz, M.R., Klein, R.I. and McKee, C.F. (2007) Molecular Line Emission from Massive Protostellar Disks: Predictions for ALMA and EVLA. The Astrophysical Journal, 665, 478. http://dx.doi.org/10.1086/519305

[4] Bonnell, I.A., Bate, M.R., Clarke, C.J. and Pringle, J.E. (2001) Competitive Accretion in Embedded Stellar Clusters. MNRAS, 323, 785-794. http://dx.doi.org/10.1046/j.1365-8711.2001.04270.x

[5] Bonnell, I.A. and Bate, M.R. (2002) Accrection in Stellar Clusters and the Collisional Formation of Massive Stars. MNRAS, 336, 659-669. http://dx.doi.org/10.1046/j.1365-8711.2002.05794.X

[6] Bonnell, I.A. and Bate, M.R. (2006) Star Formation through Gravitational Collapse and Competitive Accretion. MNRAS, 370, 488.

[7] Bonnell, I.A., Bate, M.R. and Vine, S.G. (2003) The Hierarchical Formation of a Stellar Cluster. MNRAS, $343,413$. http://dx.doi.org/10.1046/j.1365-8711.2003.06687.x

[8] Moscadelli, L., Reid, M.J., Menten, K.M., et al. (2009) Trigonometric Parallaxes of Massive Star Forming Regions. II. Cep A and NGC 7538. The Astrophysical Journal, 693, 406. http://dx.doi.org/10.1088/0004-637X/693/1/406

[9] Gibb, E.L., Whittet, D.C.B., Boogert, A.C.A. and Tielens, A.G.G.M. (2004) Interstellar Ice: The Infrared Space Observatory Legacy. The Astrophysical Journal, 151, 35. http://dx.doi.org/10.1086/381182

[10] van der Tak, F.F.S., van Dishoeck, E.F. and Caselli, P. (2000) Abundance Profiles of $\mathrm{CH}_{3} \mathrm{OH}$ and $\mathrm{H}_{2} \mathrm{CO}$ toward Massive Young Stars as Tests of Gas-Grain Chemical Models. A\&A, 361, 327.

[11] Lacy, J.H., Richter, M.J., Greathouse, T.K., Jaffe, D.T. and Zhu, Q. (2002) TEXES: A Sensitive High-Resolution Grating Spectrograph for the Mid-Infrared. Publications of the Astronomical Society of the Pacific, 114, 153. http://dx.doi.org/10.1086/338730

[12] Barentine, J.C. and Lacy, J.H. (2012) A Comparative Astrochemical Study of the High-Mass Protostellar Objects NGC 7538 IRS 9 and IRS 1. Astrophysical Journal, 757, 111.

[13] Ulrich, B.L. and Haas, R.W. (1976) Absolute Calibration of Millimeter-Wavelength Spectral Lines. Astrophysical Journal Supplement Series, 30, 247-258. http://dx.doi.org/10.1086/190361

[14] Doty, S.D., van Dishoeck, E.F., ven der Tak, F.F.S. and Boonman, A.M.S. (2002) Chemistry as a Probe of the Structures and Evolution of Massive Star-Forming Regions. Astronomy \& Astrophysics, 389, 446-463. http://dx.doi.org/10.1051/0004-6361:20020597

[15] Doty, S.D. and Neufeld, D.A. (1997) Models for Dense Molecular Cloud Cores. Astrophysical Journal, 489, 122.

[16] Gonzalez-Alfonso, E. and Cernicharo, J. (1997) Explanation of 29SiO, 30SiO, and High-v 28SiO Maser Emission. Astronomy \& Astrophysics, 322, 938.

[17] Visser, R., Kristensen, L.E., Bruderer, S., van Dishoeck, E.F., Herczeg, G.J., Brinch, C., et al. (2012) Modeling Herschel Observations of Hot Molecular Gas Emission from Embedded Low-Mass Protostars. Astronomy \& Astrophysics, 537, Article No. A55. http://dx.doi.org/10.1051/0004-6361/201117109

[18] Boogert, A.C.A., Helmich, F.P., van Dishoeck, E.F., Schutte, W.A., Tielens, A.G.G.M. and Whittet, D.C.B. (1998) The Gas/Solid Methane Abundance Ratio toward Deeply Embedded Protostars. Astronomy \& Astrophysics, 336, 352.

[19] Jacquinet-Husson, N., Scotta, N.A., Chédina, A., Garcerana, K., Armantea, R., Chursinb, A.A., et al. (2005) The 2003 Edition of the GEISA/IASA Spectroscopic Database. Journal of Quantitative Spectroscopy and Radiative Transfer, 95, 429-467. http://dx.doi.org/10.1016/j.jqsrt.2004.12.004

[20] Moré, J.J., Garbow, B.S. and Hillstrom, K.E. (1980) User Guide for MINPACK-1. Argonne National Laboratory Report ANL-80-74, Argonne, Ill.

[21] Doty, S.D. and Palotti, M.L. (2002) A Study of Some Current Methods of Analysing Observations of Star-Forming Regions. Monthly Notices of the Royal Astronomical Society, 335, 993-1004. http://dx.doi.org/10.1046/j.1365-8711.2002.05681.x

[22] Bruderer, S., Doty, S.D. and Benz, A.O. (2009) Chemical Modeling of Young Stellar Objects, I. Method and Benchmarks. Astrophysical Journal Supplement Series, 183, 179-196.

[23] Mitchell, G.F., Curry, C., Maillard, J.P. and Allen, M. (1989) The Gas Environment of the Young Stellar Object GL 2591 Studied by Infrared Spectroscopy. Astrophysical Journal, 341, 1020-1034. http://dx.doi.org/10.1086/167560

[24] Mitchell, G.F., Maillard, J.P., Allen, M., Beer, R. and Belcourt, K. (1990) Hot and Cold Gas toward Young Stellar Objects. Astrophysical Journal, 363, 554-573. http://dx.doi.org/10.1086/169365

[25] Fraser, H.J., Collings, M.P., McCoustra, M.R.S. and Williams, D.A. (2001) Thermal Desorption of Water Ice in the Interstellar Medium. Monthly Notices of the Royal Astronomical Society, 327, 1165-1172. http://dx.doi.org/10.1046/j.1365-8711.2001.04835.x

[26] Collings, M.P., Anderson, M.A., Chen, R., Dever, J.W., Viti, S., Williams, D.A. and McCoustra, M.R.S. (2004) A Laboratory Survey of the Thermal Desorption of Astrophysically Relevant Molecules. Monthly Notices of the Royal Astronomical Society, 354, 1133-1140. http://dx.doi.org/10.1111/j.1365-2966.2004.08272.x 
[27] Collings, M.P., Dever, J.W., Fraser, H.J., McCoustra, M.R.S. and Williams, D.A. (2003) Carbon Monoxide Entrapment in Interstellar Ice Analogs. Astrophysical Journal, 583, 1058-1062. http://dx.doi.org/10.1086/345389

[28] Hasegawa, T.I., Herbst, E. and Leung, C.M. (1992) Models of Gas-Grain Chemistry in Dense Interstellar Clouds with Complex Organic Molecules. Astrophysical Journal Supplement Series, 82, 167-195. http://dx.doi.org/10.1086/191713

[29] Brooke, T.Y., Tokunaga, A.T., Weaver, H.A., Crovisier, J., Bockelée-Morvan, D. and Crisp, D. (1996) Detection of Acetylene in the Infrared Spectrum of Comet Hyakutake. Nature, 383, 606-608. http://dx.doi.org/10.1038/383606a0

[30] Moore, M.H. and Hudson, R.L. (2005) Astrochemistry: Recent Successes and Current Challenges. Lis, D., Blake, G. and Herbst, E., Eds., Cambridge University Press, Cambridge, 247-260.

[31] Tielens, A.G.G.M. and Charnley, S.B. (1997) Circumstellar and Interstellar Synthesis of Organic Molecules. In: Whittet, D.C.B., Ed., Planetary and Interstellar Processes Relevant to the Origins of Life, Kluwer Academic Publishers, Dordrecht, 23.

[32] Knez, C., Lacy, J.H., Evans, N.J., Richter, M.J., Boonman, A.M.S. and van Dishoeck, E.F. (2003) The Study of Interstellar Chemistry through Mid-Infrared Spectroscopy. RevMexAA (Serie de Conferencias), 18, 45-47.

[33] Mueller, K.E., Shirley, Y.L., Evans II, N.J. and Jacobson, H.R. (2002) The Physical Conditions for Massive Star Formation: Dust Continuum Maps and Modeling. Astrophysical Journal Supplement Series, 143, 469-498.

[34] Bruderer, S., Benz, A.O., Bourke, T.L. and Doty, S.D. (2009) Evidence of Warm and Dense Material along the Outflow of a High-Mass YSO. Astronomy \& Astrophysics, 503, L13-L16. http://dx.doi.org/10.1051/0004-6361/200912620

[35] Bruderer, S., Benz, A.O., Doty, S.D., van Dishoeck, E.F. and Bourke, T.L. (2009) Multidimensional Chemical Modeling of Young Stellar Objects. II. Irradiated Outflow Walls in a High-Mass Star-Forming Region. Astrophysical Journal, 700, 872.

[36] Bruderer, S., Benz, A.O., Stäuber, P. and Doty, S.D. (2010) Multidimensional Chemical Modeling of Young Stellar Objects. III. The Influence of Geometry on the Abundance and Excitation of Diatomic Hydrides. Astrophysical Journal, 720, 1432.

[37] Sandell, G., Goss, W.M. and Wright, M. (2005) Protostars and Outflows in the NGC 7538 IRS 9 Cloud Core. Astrophysical Journal, 621, 839-852.

[38] Peretto, N., Fuller, G.A., Duarte-Cabral, A., Avison, A., Hennebelle, P., Pineda, J.E., et al. (2013) Global Collapse of Molecular Clouds as a Formation Mechanism for the Most Massive Stars. Astronomy \& Astrophysics, 555, Article No. A112. http://dx.doi.org/10.1051/0004-6361/201321318

[39] Sánchez-Monge, A., Beltrán, M.T., Cesaroni, R., Etoka, S., Galli, D., Kuma, M.S.N., et al. (2014) A Necklace of Dense Cores in the High-Mass Star Forming Region G35.20-0.74N: ALMA Observations. http://www.arxiv.org/pdf/1406.4081v1.pdf

[40] Krumholz, M.R., Klein, R.I., McKee, C.F., Offner, S.S.R. and Cunningham, A.J. (2009) The Formation of Massive Star Systems by Accretion. Science, 323, 754-757. http://dx.doi.org/10.1126/science.1165857

[41] Fallscheer, C., Beuther, H., Sauter, J., Wolf, S. and Zhang, Q. (2011) A High-Mass Dusty Disk Candidate: The Case of IRAS 18151-1208. Astrophysical Journal, 729, 66.

[42] Doty, S.D., van Dishoeck, E.F. and Tan, J.C. (2006) Astrochemical Confirmation of the Rapid Evolution of Massive YSOs and Explanation for the Inferred Ages of Hot Cores. Astronomy \& Astrophysics, 454, L5-L8. http://dx.doi.org/10.1051/0004-6361:20065320

[43] Woitke, P., Kamp, I. and Thi, W.F. (2009) Radiation Thermo-Chemical Models of Protoplanetary Disks. I. Hydrostatic Disk Structure and Inner Rim. Astronomy \& Astrophysics, 501, 383-406. http://dx.doi.org/10.1051/0004-6361/200911821 
Scientific Research Publishing (SCIRP) is one of the largest Open Access journal publishers. It is currently publishing more than 200 open access, online, peer-reviewed journals covering a wide range of academic disciplines. SCIRP serves the worldwide academic communities and contributes to the progress and application of science with its publication.

Other selected journals from SCIRP are listed as below. Submit your manuscript to us via either submit@scirp.org or Online Submission Portal.
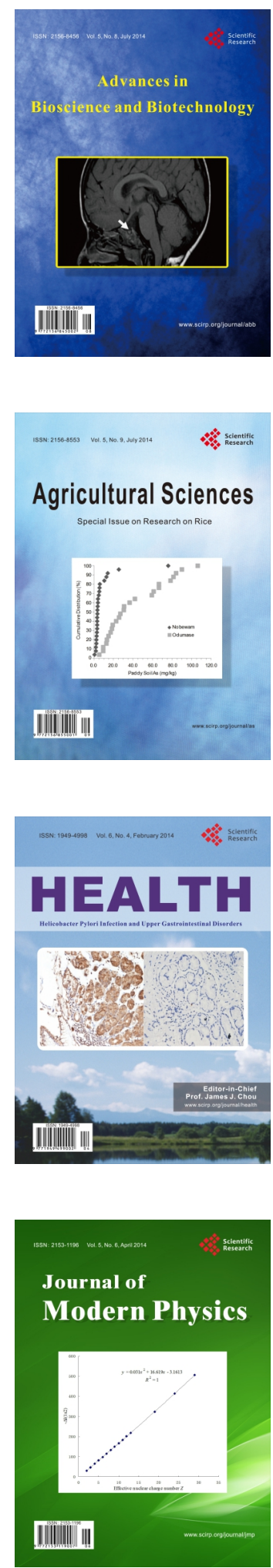
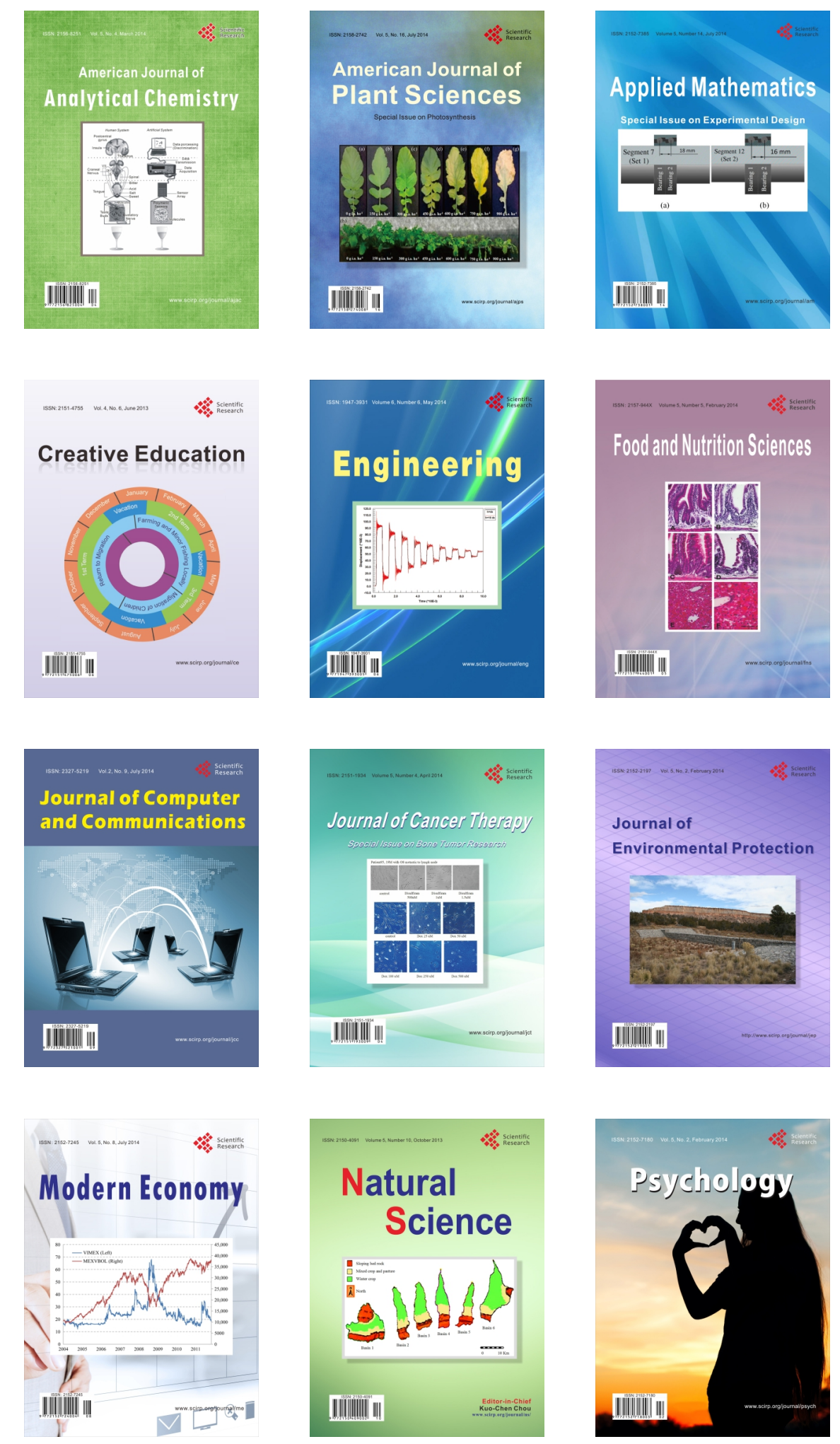